\title{
Using an Intelligent Tutoring System to Support Learners' WMC in e-learning: Application in Mathematics Learning
}

\author{
https://doi.org/10.3991/ijet.v13i12.8938
}

\author{
Bouchra El Mamoun $^{(\bowtie)}$, Mohamed Erradi, Abderrahim El Mhouti \\ Abdelmalek Essaadi University, Tetouan, Morocco \\ elmamounbouchra@gmail.com
}

\begin{abstract}
Learning is a complex process linked to a number of individual, cognitive and affective characteristics of the learner. In this context, the learner's Working Memory (WM) concept has also been identified as a preacher of learning performance. In the learning process, solving a problem is an essential part of the learner's WM. Its capacity and performance are predictive factors of academic success. The success of the given problem depends on a good selection, processing and transformation of this information. This mental work within the WM requires a good WM Capacity (WMC). The improvement of the WMC needs some strategies which focus on the learning content and the use of educational technologies in the learning process.

Encouraged by the results of previous research, this work aims to harness the potential of educational technologies to enhance the learners' WM ability in learning mathematics in an e-learning environment. In order to help learners with low WMC in e-learning, this paper proposes to set up an e-learning platform integrating an Intelligent Tutorial System (ITS). The purpose of this ITS is to manage the limited ability of the learner's WM by adopting strategies that improve the transition of mathematical knowledge from short-term memory to long-term memory. The proposed ITS generates e-learning content that will serve to improve the learner's WMC, and subsequently improve his learning performance.
\end{abstract}

Keywords-Working Memory, Working Memory Capacity, e-learning, ITS.

\section{Introduction and research issues}

Learning is a continuous process of acquiring new or modifying knowledge. The learning process is related to a number of individual, cognitive and affective characteristics that explain the corresponding variability in learning performance. In this context, the concept of Working Memory (WM) has been identified as a preacher of learning performance. In the learning process, solving a problem is an essential part of the learner's WM. Its capacity and performance are predictive factors of academic success. It has a significant impact on the learner's performance [1].

Indeed, when solving a problem by a learner, his WM receives essentially two types of information: information that comes from the school environment, selected by the 
student's sensory receptors, and information already stored in its Long Term Memory (LTM), relevant and useful for the realization of the problem requested. The success of the given problem depends on a good selection, processing and transformation of this information. This mental work within the WM requires a good WM Capacity (WMC).

To improve the learner's WMC, several strategies have been put in place [2]. These strategies focus on the learning content and the use of educational technologies in the learning process in order to promote the WMC management and thus improve the student's academic performance thereafter [1].

Encouraged by the results of previous research, this work aims to harness the potential of educational technologies to enhance the learners' WM ability in learning mathematics in an e-learning environment.

Thus, in order to help learners with low WMC in e-learning, this article proposes to set up an e-learning platform integrating an Intelligent Tutorial System (ITS). The purpose of this ITS is to manage the limited ability of the learner's WM by adopting strategies that improve the transition of mathematical knowledge from Short-Term Memory (STM) to LTM. The proposed ITS generates e-learning content that will serve to improve the learner's WMC, and subsequently improve his or her academic performance.

The design of the proposed ITS takes into account the different characteristics of the WM, which allows the ITS to present to the learner the pure information that it can code, organize and develop effectively, promotes the acquisition of knowledge.

In addition to the basic models (domain, tutor, learner and interface), the ITS architecture incorporates a "WMC management model". This model, in permanent interaction with the other models, allows, as a first step, to test the learner's WMC as well as his level of information processing in order to group a database describing the learner's profile. The results of the diagnosis will be used to classify the WMC and the level of information processing that the learner can achieve at intervals well defined by the ITS. Based on these findings, the ITS directs the learner to learning activities, be it improvement activities of their WMC (eg WMC training activities) if their results are too low, or mathematical problems to solve, appropriate to his skills, and allow a transition from one level to a higher one, as he succeeds in the proposed problems.

The rest of this paper is structured as follows: first, the paper presents a literature review of the main concepts on which this work is based, namely, WM, e-learning and ITSs. Secondly, this work exploits the characteristics of ITSs and those of the WM to design an ITS that responds to the problematic, explaining the vision about the improvement of the WMC via the targeted ITS, and subsequently ensure a better academic performance of the learner, user of this ITS. This research ends with a conclusion and perspectives.

\section{Theoretical background}

\subsection{Working memory}

WM: definition and models. WM is a model derived from the current "Information Processing System (IPS)" [3] for which the abstract and symbolic information is treated 
in a localized way and sequentially. Working memory allows you to temporarily retain and manipulate new information that is involved in processes such as comprehension, learning and reasoning. This model is proposed by [4] as an alternative to the concept of short-term memory of the modal model proposed by [5].

The most adopted model of WM [6] is the tripartite model consisting of : the executive center, representing an attention control system capable of supervising the information from two enslaved systems and, the phonological loop and the visuo-spatial sketchpad. The phonological loop processes information from language and hearing, while the visual-spatial register holds images.

In 2005, a new component of the WM was added [7] to its initial model called "the episodic buffer", which response to a number of experimental facts not predicted by the tripartite model (Figure1).

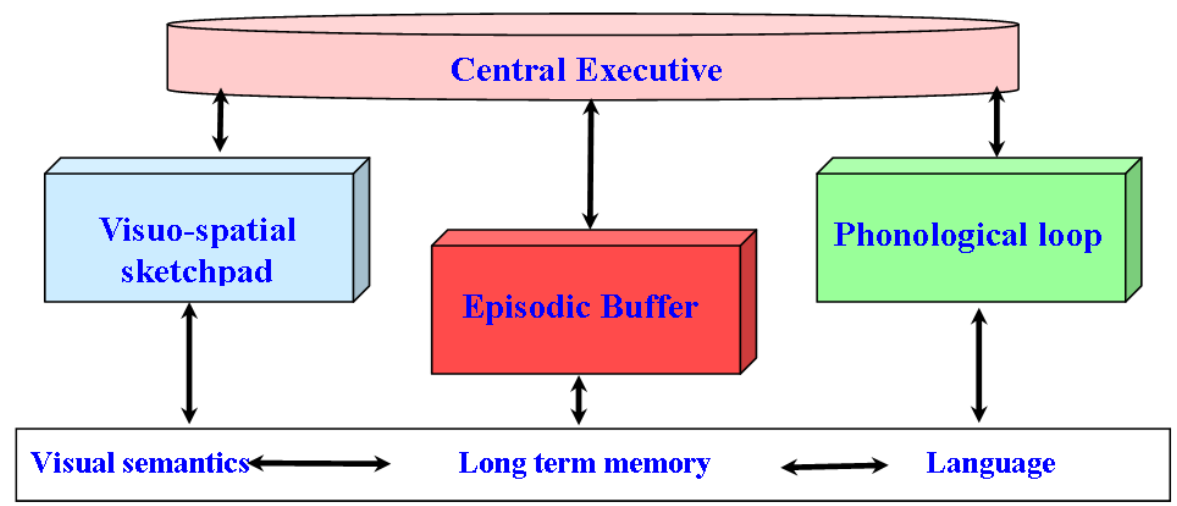

Fig. 1. Working memory model according [7]

WMC. The WMC is limited. In 1956, the ability of an adult's working memory was determined [8] to 7 items $+/-2$. This means that one would have the ability to jointly manage 7 isolated $+/-2$ elements before that these do not fade, replaced by others. This ability is expressed by the working memory span, which is the number of isolated items that can be stored in memory for up to one minute. It is measured by many tests.

Reference [9] hypothesized that WMC "is about using attention to maintain or suppress information". The WMC does not really concern storage or memory as such, but rather the capacity for sustained, controlled attention to interference or distraction [10].

2.2 E-learningE-learning: overview. E-learning is the use of technology to enable people to learn anywhere and anytime. It covers all training methods using the computer tool. This definition includes several independent axes:

- Online or offline media

- Individual or collective learning

- Trainer present, remote or absent 
When we talk about e-learning, other terms are sometimes used, such as : online formation, online learning, online education, distance education or distance learning.

In itself, e-learning does not imply anything about: the physical presence of a trainer, the very existence of the trainer, the autonomy of the training, or the place of training.

In practice, e-learning is often considered as the means to follow a training from any computer station equipped with an Internet connection.

e-learning covers all types of training from an interactive DVD-ROM to a virtual classroom with a trainer and co-learners.

E-learning environments. The e-learning environment refers to the collaborative interactions used for knowledge acquisition within the online computer-mediated digital system [11]. There are different e-learning environment namely:

- A learning management system (LMS): is a software application or Web-based technology used to plan, implement, and assess a specific learning process. Typically, a LMS provides an instructor with a way to create and deliver content, and students with the ability to use interactive features such as discussions, video conferencing, and forums. [12]

- A Learning Content Management System (LCMS): is a tool or software that allows an organization, university, or company to create and publish learning content and publish it in print and web formats. Good LCMS will also have analytics so you can see data on how learners are interacting with your content, and some offer a singlesource input, which allows creating the content.

- A Virtual Learning Environment (VLE): is a system for delivering learning materials to students via the web. These systems include assessment, student tracking, collaboration and communication tools. For those who edit them, VLEs may have a de facto role as authoring and design environments. [13] VLEs have been adopted by almost all higher education institutions in the English-speaking world.

Managing the WMC in e-learning: mains strategies. Working memory is the mechanism that manipulates information temporary located in the short-term memory and either discards it or facilitates its transition to the LTM e-Learning content should be based on strategies that enhance this transition in order to guarantee that learning has actually taken place.

As working memory has a limited capacity and duration, it tries, when there is much incoming information, to retain only the meaningful one and discard all the rest to make room for more incoming information. Based on this there are certain strategies eLearning professionals should follow in order to manage working memory's limited capacity. Previous research [14] cites some of these strategies as:

- Present new concepts starting from simplest to most complex;

- Limit the number of distractions ;

- Provide opportunities for practice;

- Chunking;

- Present information in meaningful and easily digestible ways;

- Include assignments that activate long-term memory schemata; 


\subsection{Intelligent Tutoring Systems (ITS)}

Description. An intelligent tutorial system (ITS) can be defined as a computerbased learning environment that integrates computational models borrowed from cognitive science, computational linguistics, education science, artificial intelligence, mathematics, and computer science to other areas [15]. This system is characterized by the fact that it makes it possible to automate teaching and to promote learning without the intervention of a human tutor.

ITSs have been proven over time and have been very effective, particularly in the fields of mathematics, science and technology [16]. They represent a learning medium that has a much greater impact than the classroom environment and can capture the attention and interest of the learner for hours.

Basic architecture and operation. The classic conceptual architecture of an ITS consists of four models:

- A domain model or expert model: which knows what to teach

- A tutor model or pedagogical model: which knows how to teach

- A learner model: which personalizes the learner

- A model of the interface: which presents my visible means allowing interrelationship between the learner and the system.

Figure 2 presents the different classical models of an ITS and the interactions between them [17]:

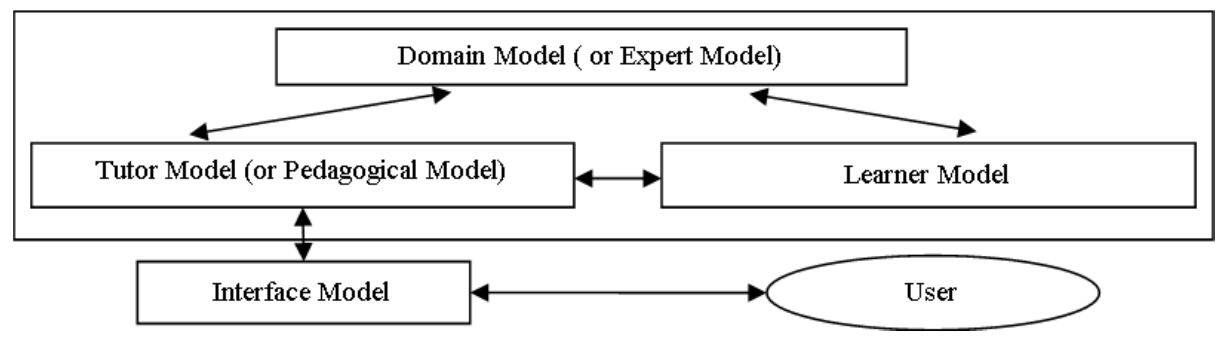

Fig. 2. The basic conceptual architecture of an ITS

An ITS is able to track learner behavior using a process called learner modeling. The learner model can include knowledge of the learning subject, skills, learning strategy, level of motivation, emotion, and other attributes that can be assessed. The ITS uses information from this model to interact with the learner adaptively.

Learning content models specify "what" to teach using knowledge bases as well as teaching strategies that specify "how" to teach it. They make inferences about the learning object and the activities that need to be done by a learner in order to dynamically adapt the content or the style of training offered. In this sense, ITSs are different from traditional learning systems by the good determination of domain knowledge, pedagogical knowledge and learner representation.

ITS and WMC improvement. Research [18] has shown that WM is a key factor in online learning. Learning in a hypermedia environment requires cognitive processing 
of visual and verbal content, involving both the slave systems of the WM, and the resources of the executive office. As a result, WMC can predict learning performance in hypertext environments, hence the importance of measuring this ability before starting e-learning. This requires certain structures or methods of presenting content to be learned, as well as strategies and techniques that take into consideration the WMC and the level of manipulation and processing of information within the learner's WM.

In this respect, ITSs are presented as a tool that brings together the need to describe a profile of the learner's WM, and the choice of activities appropriate to his profile, while improving his WMC by moving from a mathematical problem. to another, more data and a higher level of information processing than the problem that precedes it.

\section{Towards an ITS supported WMC model to enhance learners' performance}

\subsection{Global overview}

The success of a mathematical problem depends on a good selection, processing and transformation of its data as well as the knowledge that this problem must appeal. This success requires the application of certain strategies focused on the learning content, namely the statement of the mathematical problem as well as the knowledge and the knowledge of the student about the given learning situation.

The targeted ITS brings together the factors responsible for the success of a mathematical problem while improving the WMC of the user-user of the system. The ITS to be designed must be able to:

- Diagnose the learner knowledge and skills based on principles rather than pre-programmed responses;

- Decide on the next task to be performed

- Adapt the proposed learning situations to the learner's skills

- Ensure effective communication with the learner

- Encourage reflection, processing and coding of information by the learner

- Allow a good exploitation of the WMC of the learner

\subsection{Design of the ITS-supported WMC}

The ITS targeted organize the linked steps that the learner has to go through to enable the system to group a descriptive database of the learner's profile. This sequence consists of passing the WMC test which measures the working memory capacity, and the IPL test which measures the level of information processing within the WM. The results of these tests allow the ITS to direct the learner towards the activities he needs, whether it is improvement activities of his WMC (for example the training activities of the WMC) if his results are too weak., or mathematical problems to solve, appropriate to his skills, and allow a transition from one level to a higher one, as he succeeds the proposed problem. These steps are presented in Figure 3. 
In principle, it is the "Answer the submitted problem" phase that makes the difference between the ITS to be designed and the other ITSs dedicated to school learning. Indeed, this phase allows the learner to identify and then organize the data within the WM, which decreases the memory load within this memory, which allows a good exploitation of the WMC. In addition, this phase ensures the back and forth chained and structured between the STM and the LTM, and an organized presentation, at the level of the WM, of the different data collected within these two memories.

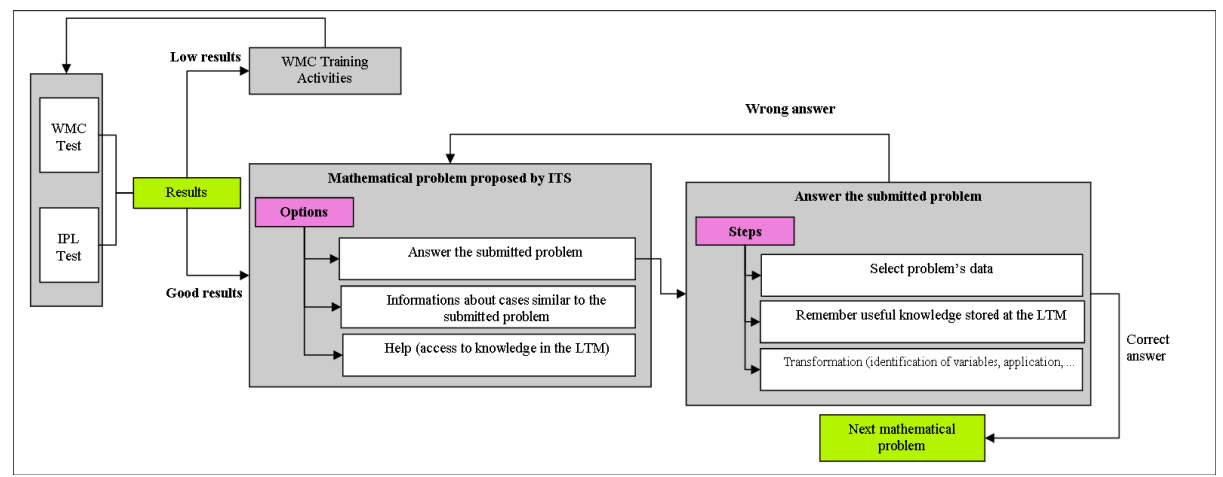

Fig. 3. The overall design of the ITS targeted

The success of these steps allows the evaluation of the competences of the learner at the level of the selection, organization, coding and elaboration of the information that one asks him when solving the mathematical problem proposed. It also allows maximum exploitation of the WMC of the learner, and leads him to practice the basic techniques to succeed a mathematical problem.

If the learner realizes the mathematical problems proposed, one after the other, respecting the proposed stages of this phase, he will take the habit of thinking and act in the same way when solving problems even in class (without tools technological). This is where we can ensure an improvement of the WMC, and later that of the student's academic performance.

\subsection{Functioning of ITS supported WMC}

An ITS works by selecting and presenting a problem or question to the learner. It compares the desired solution to that provided by the learner and then performs a diagnosis based on the differences found between the two solutions. After providing adequate feedback to the learner, the system makes a new assessment, updates the learner information, and repeats the cycle.

At the first contact between the target ITS and the user learner, the ITS proposes to the user to pass:

- $\boldsymbol{A} \boldsymbol{W M C}$ test: is a test that allows the measurement of numerical memory and in letters. The results of this test are used to rank the learner's WMC at one of the capacity's intervals determined in the ITS. This interval is the first variable that the 
ITS takes into consideration when proposing the mathematical problem to be solved. It directs the learner towards problems whose number of data necessary to its realization does not exceed the WMC of this learner.

- An IPL test: is a test of measurement of the level of processing of the information that the learner can reach the resolution of the mathematical problems. It is a set of learning situations that are based on the processing of a set of information, in order to answer specific questions. The results of this test are used to classify the level of information processing that the learner can achieve in one of the intervals determined within the ITS. This interval is the second variable that the ITS takes into consideration when proposing mathematical problems to be solved. It directs the learner to problems that respect the learner's ability to make transformations and treatments when solving the mathematical problems chosen by the ITS.

\subsection{ITS architecture}

The conceptual architecture of an ITS cited above will serve as the basis for developing the ITS target. However, in trying to achieve the intended objectives of this research, another model called "WMC Management Model" is proposed, which ensures the planned tasks of the ITS to be designed. The conceptual architecture of the ITS targeted is illustrated in Figure 4.

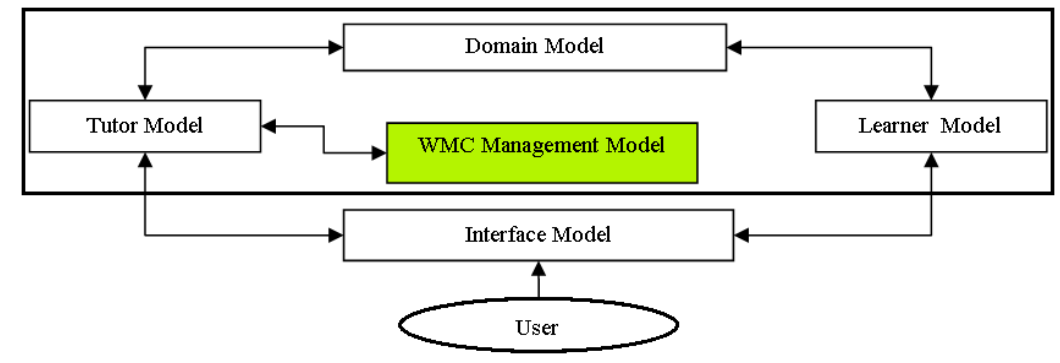

Fig. 4. Conceptual Architecture of the ITS targeted

The architecture of the component models of the targeted ITS is based on various research results made concerning ITSs dedicated to the improvement of learning, specifically mathematics, and also those interested in mathematical problem solving processes, in addition to research that focuses on strategies for developing WMC.

Interface Model. This component is the input of the ITS, which provides the learner with an environment with which he interacts, by giving and receiving messages from the system. It can be presented in different forms depending on the learning environment chosen and the intended purpose.

- Graphical interface: buttons, icons and menus

- Tangible interface: a command language or natural language 
This research favor that the targeted ITS will use a graphical interface, to guarantee a simple and direct use of ITS offers. The model of the interface will be presented to the learners in the form of a web interface.

Learner Model. This model is built into the system. It represents the cognitive, affective, psychological and emotional state of the learner at a given moment of his intended learning.

The construction of this model is done by comparing the performance of the learner to that of the expert, this for the same problem. We favor adopting a model composed of expert knowledge, learner knowledge and shared knowledge. This model will allow us to discover a margin of errors that the learner can commit, which will facilitate the choice of methods and techniques of remediation. The architecture of the targeted learning model is presented in the following figure:

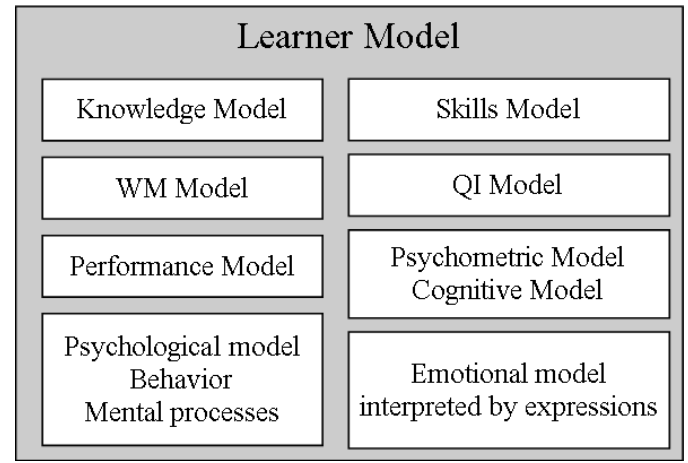

Fig. 5. The architecture of the Learner Model targeted

Tutor Model. This is the most important component of an ITS, since the other components of the system access this model to achieve their respective functions. This model is based on the domain model for planning learning activities and preparing for their implementation. Its operation consists of:

- Select the tasks to be performed by the learner according to his needs.

- Plan activities to present to the learner

- Provide the appropriate explanations

- Determine when and how to intervene when learning

- Help, accompany and guide the learner in his learning task

The architecture of the tutor model must make use of the contents of the domain, the different didactic, pedagogical, metacognitive strategies and those which mobilize the WM. The functioning of this model is based on appropriate educational approaches to make decisions in given learning situations. The tutor model targeted is illustrated in Figure 6. 


\begin{tabular}{|c|c|}
\hline $\begin{array}{l}\text { Content } \\
\text { - Domain content } \\
\text { - Content related to solving } \\
\text { mathematical problems }\end{array}$ & $\begin{array}{l}\text { Strategies mobilizing WM } \\
\text { - Training } \\
\text { - Reduce cognitive charge of WM }\end{array}$ \\
\hline $\begin{array}{l}\text { Diagrams representing the } \\
\text { problems } \\
\text { - Stimulate reflexivity } \\
\text { - To be able to supervise and } \\
\text { evaluate his actions during the } \\
\text { resolution of the given } \\
\text { problem } \\
\text { - Stimulate self-regulation }\end{array}$ & $\begin{array}{l}\text { Problem area }=\text { Research area } \\
\text { - Attempts at solution testing } \\
\text { - Defined by: } \\
\Rightarrow \text { Initial state } \\
\Rightarrow \text { Goal to reach } \\
\Rightarrow \text { Operators (actions, means ...) } \\
\Rightarrow \text { Constraints of the application } \\
\text { of the operators }\end{array}$ \\
\hline Problem types & Cognitives processes \\
\hline $\begin{array}{l}\text { Tools and methods for solving } \\
\text { mathematical problems }\end{array}$ & Teaching techniques \\
\hline
\end{tabular}

Fig. 6. The architecture of Tutor Model targeted

- Sub-model "Content": This is the body of knowledge of the field in terms of concepts, principles and procedures. This database allows the construction of activities and mathematical problems proposed.

- Sub-model "Diagrams representing the problems": This sub-model help to reduce the mental load of the WM, and to ensure the modelling of the problem given by an identified schema.

- Sub-model "Strategies are mobilizing WM": These are the set of strategies that can improve the capacity of the WM, such as training, or that can reduce the cognitive charge imposed on the WM. It proposes to adopt the strategy of mobilization and development of WM schematized by the following process:

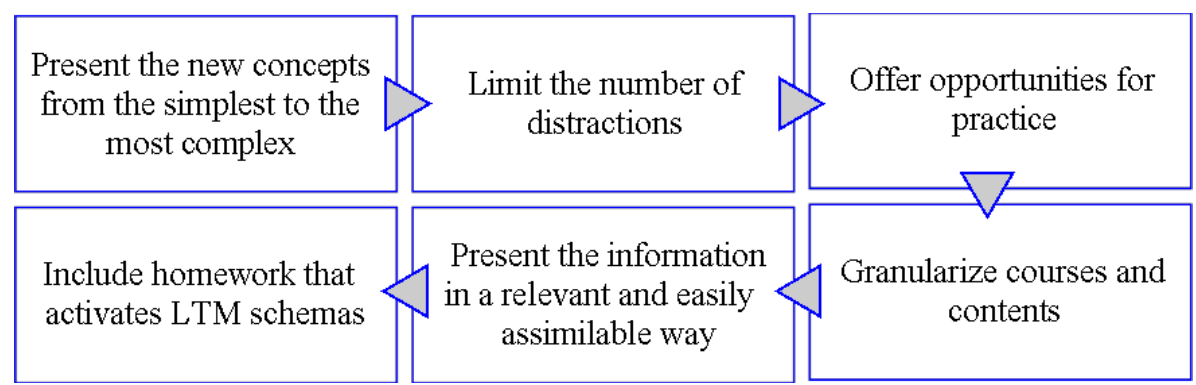

Fig. 7. The proposed strategy of mobilization and development of WM

- Sub-model "Problem area": This space allows the learner to proceed with the resolution of the given mathematical problem, and to have attempteds to test the solution. 
- Sub-model "Problem types": It is a varied series of open and complex problems, presented in various formats (articles, tables, drawings, ...). It is a sub model that offers coaching and support to the learner when solving the mathematical problem.

- Sub-model "Pedagogical techniques": This sub-model offers various pedagogical techniques, according to a model based on the training of sequences of activities. This involves several pedagogical principles like stimulation of active and constructive learning, the creation of opportunities for collaboration, and development of self-regulation of learning

- Sub-model "Tools and methods for solving mathematical problems": Structured methods are used to identify and solve a mathematical problem, and the specific tools implemented within the applied method.

- Sub-model "Cognitive process": It is the set of mental processes that allow an individual to acquire, process, store and use information or knowledge.

Domain Model. The domain model, or expert model, consists of a knowledge base that the system attempts to teach to the learner. It contains coded domain knowledge and mechanisms related to the exploitation of this knowledge. He is able to manipulate and explain the stored knowledge. It can also reason with generalized knowledge, and draw valid conclusions that are not explicitly encoded. The architecture of the domain model is presented by the following figure:

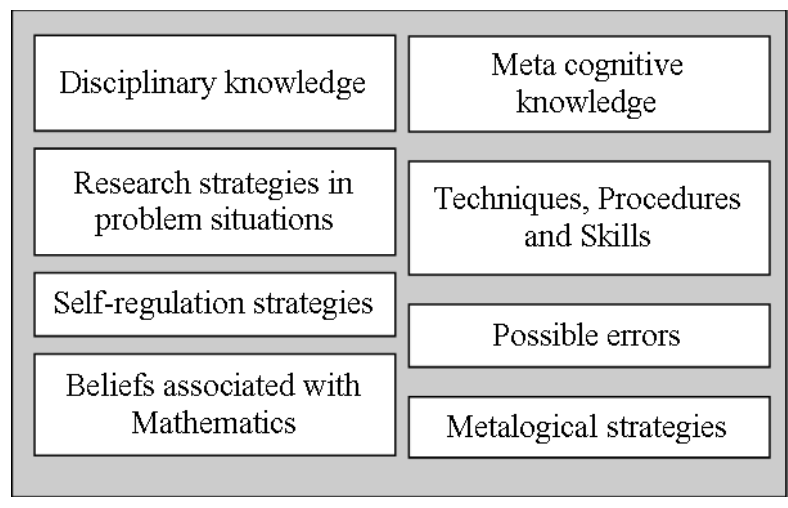

Fig. 8. The architecture of the Domain Model targeted

- Sub-model "Disciplinary Knowledge": consists of the table of contents of Mathematics as a subject, mathematical concepts, algorithms and symbols used in solving a mathematical problem.

- Sub-model "Research strategies in problem situations": these strategies introduce a systematic approach to the task at hand, and increase the probability of finding the right solution.

- Sub-model "Self-regulation strategies": these strategies are based on the four key points of self-regulation: goal-setting, planning, control and adjustment.

- Sub-model "Metalogical strategies": allow the management of meta-structures of reasoning procedures when solving mathematical problems. 
- Sub-model "Meta-cognitive Knowledge": groups the learner's abilities, the characteristics of the tasks to be carried out, as well as the validity of the manipulated strategies.

- Sub-model "Techniques, Procedures and Skills": groups the techniques, procedures and skills used to solve a mathematical problem.

- Sub-model "Possible errors": contains all the errors that the learner may commit when solving the proposed mathematical problem. The existence of this error field allows the system to choose the appropriate remediation method in order to overcome the difficulties that arise in this resolution.

- Sub-model "Beliefs Associated with Mathematics" : this is the set of Beliefs related to the learner's own relationship to learning and problem solving, as well as beliefs related to the social context in which the mathematical activities take place, as well as beliefs about Mathematics and solving problems themselves.

WMC Management Model. This model takes a database of the learner by measuring his WMC. According to the results found, the system guides the learner to the appropriate activities at his WMC. It consists of two sub models:

- "WMC tests": who will test the ability of the WM for each learner, as well as its level of handling and processing information within the WM

- "WMC Improvement": responsible for improving the functioning of the WM by using strategies mobilizing this memory

The architecture of the "Management of the WMC" Model is represented by the following figure:

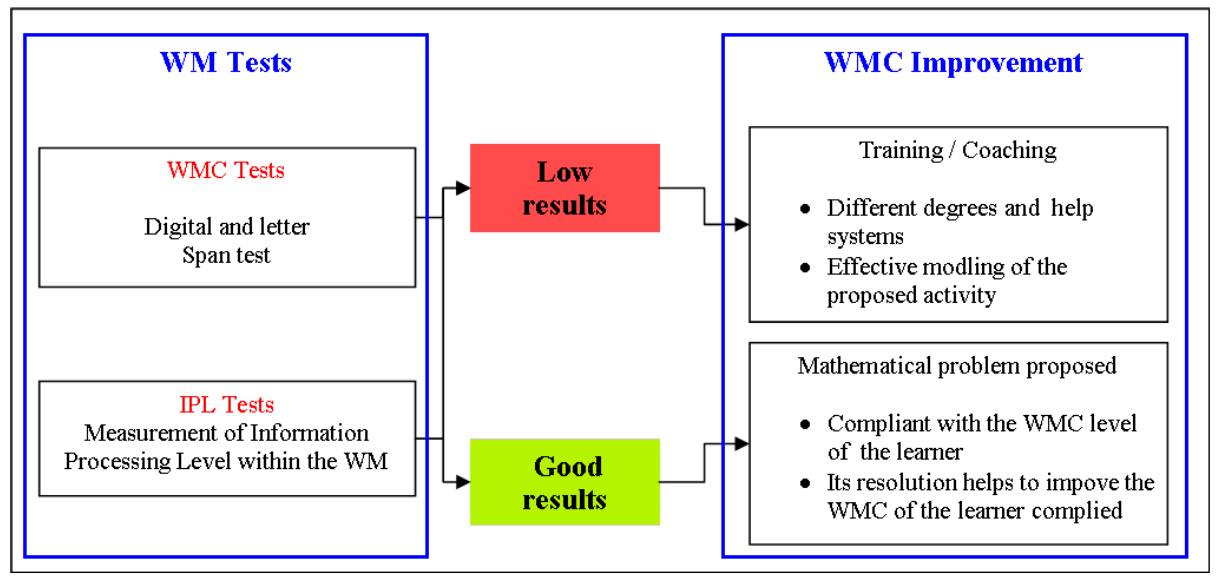

Fig. 9. The architecture of the WMC Managemet Model targeted

The general architecture of the targeted ITS. All the proposed models are grouped in the ITS architecture covered by the following schema: 


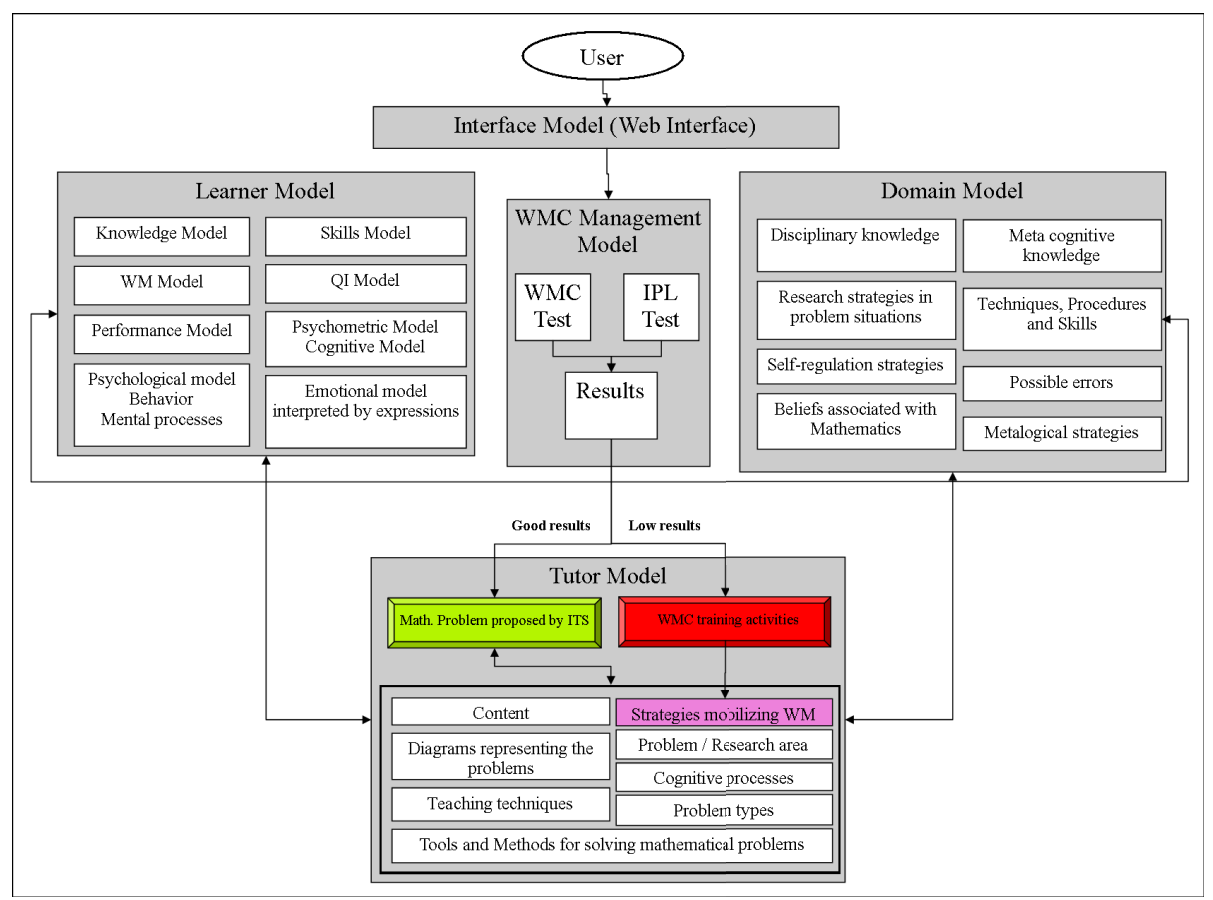

Fig. 10. The general architecture of the ITS targeted

The ITS interface ensures contact between the learner and the various models of the system. Interactions between ITS models are favoured as the learner moves from presented content from one interface to another. In the light of the results collected by the learner in each stage of the proposed work, the ITS uses the different knowledge, data, techniques, procedures and skills that the learner must manipulate in order to succeed in the mathematical problem proposed

\section{Summary and future work}

Without any doubt, working memory is one of the most important brain functions with a significant impact on the learning process. When considering how working memory functions in order to improve the online training experience, instructional designers should have two things in mind: how to manage working memory's limited capacity and working memory strategies that will facilitate the transition of information from short-term to LTM.

By studying the factors that influence the acquisition of learning in Mathematics, be it at the WM level and its characteristics or at the level of mathematical problem-solving procedures, the ITS targeted during this research is presented as a tool that brings together the need to describe a learner's MDT profile, and the choice of activities appropriate to his profile, while improving his WMC by moving from one mathematical 
problem to another, a number of data and a level of information treatment superior than the problem that precedes it.

Future research aims to implement the ITS designed, test it with learners and improve its architecture by adopting different strategies that appear essential for the development of the WMC of the learner, and the progress of his academic performance in Mathematics.

\section{$5 \quad$ References}

[1] El Mamoun, B, Erradi, M, El Mhouti, A. (2017). Using Digital Resources in the Meas-ure of the Impact of Working Memory on Students` Acquisition of Mathematical Knowledge. International Journal of Computer Sciences and Engineering, 5(12): 123-129.

[2] Gathercole, S, Alloway, T. (2008). Working memory and learning: A practical guide for teacher. Portland, OR : Sage.

[3] George, A. (1956). Miller The Magical Number Seven, Plus or Minus Two: Some Lim-its on Our Capacity for Processing Information. Psychological Review, 63: 81-97. https://doi.org/10.1037/h0043158

[4] Barrouill, P, Camos, V. (2014). The developpement of working memory, Psychologie francaise 59.

[5] ABUDYAR, M. (2012). La mémoire de travail et les difficultés d'apprentissage. Series of Child Assessment and Education Center, Kuwait.

[6] Baddeley, A. (1996). The fractionation of working memory",Proc. Natl. Acad. Sci. USA, 93:3468-13472. https://doi.org/10.1073/pnas.93.24.13468

[7] Baddeley, A. (2000). The episodic buffer: a new component of working memory?,Trends in Cognitive Sciences, 4(11): 417-423. https://doi.org/10.1016/S1364-6613(00)01538-2

[8] Alloway, T. P, Gathercole, S, Pickering, S. I, Child, D. (2006). Verbal and visuospatial shortterm and working memory in children: are they separable?. 77(6):1698-1716.

[9] Engle, R. W. (2002). Working memory capacity as executive attention, Curr. Dir. Psy-chol. Sci. 11: 19-23. https://doi.org/10.1111/1467-8721.00160

[10] Wilhelm, O, Hildebrandt, A, Oberauer K. (2013). What is working memory capacity, and how can we measure it?. Front Psychol. 4. https://doi.org/10.3389/fpsyg.2013.00433

[11] Mkrttchien, V, Bershadsky, A, Bozhdat, A, Kataev, M, Kataev, S. (2016). Estimation and Control Techniques in E-Learning Systems. IGI Global Book series Advances Educational Technologies and Instructional Design. https://doi.org/10.4018/978-1-4666-9489-7

[12] Rouse, M. (2017). The essential guide to managing HR technology trends. TechTarget.

[13] Liz, M. (2013). The challenge of teachers' design practice. Written at London. In Beetham, Helen; Sharpe, Rhona. Rethinking pedagogy in a digital age, 2013. Oxford: Routledge. p. 65.

[14] Pappas, C. (2015). Enhancing Working Memory: 6 Strategies for eLearning Profes-sionals. eLearning INDUSTRY. atio Graesser, A. C., Conley, M. W, Olney, A. (2012). Intelligent tutoring systems. In S. Graham, \& K. Harris (Eds.), APA Educational Psychology Handbook: Vol. 3. Appli-cations to Learning and Teaching (pp. 451-473). Washington, DC: American Psycho-logical Association.

[15] Graesser, A. C. , VanLehn, K. , Rosé, C. P., J ordan, P. W. et Harter, D. (2001). Intelli-gent tutoring systems with conversational dialogue. A I magazine, 22( 4) , 39.

[16] Nkambou, R., Mizoguchi, R. et Bourdeau, J. (2010). Advances in intelligent tuutoring systems, volume 308. Springer Science \& Business Media. https://doi.org/10.1007/978-3-642$\underline{14363-2}$ 
Paper-Using an Intelligent Tutoring System to Support Learners' WMC in e-learning: Application in...

[17] Tato, N, Adrienne, A. (2016). Développement d'un système tutoriel intelligent pour l'apprentissage du raisonnement logique. Master disertation, Montréal (Québec, Cana-da), Québec Université. Canada.

\section{Authors}

Bouchra EL MAMOUN - PhD student a in LIROSA laboratory at Abdelmalek Essaadi University, Faculty of Sciences, Tetouan, Morocco. His research fields are computer science, mathematics, e-learning, pedagogy and Web technologies.

Mohamed ERRADI - received the $\mathrm{PhD}$ degree from the Mohammed V University in Morocco. He is Professor and computer science \& pedagogy researcher in Abdelmalek Essaadi University. He is member of LIROSA laboratory. His research fields are: e-learning, pedagogy, and educational technologies.

Prof. ERRADI is currently Professor and pedagogy trainer at Ecole Normale Supérieure, Abdelmalek Essaadi University, Tetouan, Morocco. He has published several articles on e-learning and educational technologies.

Abderrahim EL MHOUTI - received the PhD degree in Computer Science in 2015 in LIROSA laboratory and the Master degree in Software Quality in 2011 from Abdelmalek Essaadi University, Faculty of Sciences, Tetouan, Morocco.

Prof. EL MHOUTI is a Professor of Computer Science at the Faculty of Sciences and Technologies of Al-Hoceima in Abdelmalek Essaadi University, Morocco. His research fields include: collaborative e-learning and pedagogy, Web 2.0 technologies, cloud computing, and Big data. He has published several articles on e-learning, Web 2.0, educational technologies and cloud computing.

Article submitted 30 May 2018. Resubmitted 06 June 2018. Final acceptance 23 November 2018. Final version published as submitted by the authors. 